\title{
Retrospective survey of the management of miliary tuberculosis in South and West Wales, 1976-8
}

\author{
RDH MONIE, AM HUNTER, KMS ROCCHICCIOLI, JP WHITE, IA CAMPBELL, \\ GS KILPATRICK
}

\begin{abstract}
From the Welsh National School of Medicine, and the Department of Thoracic Medicine, Llandough Hospital, Penarth, Glamorgan
\end{abstract}

ABSTRACT In a survey of 1000 patients with tuberculosis 28 were found to have miliary disease. Half of these patients were over 60 years old. Anorexia and weight loss were present in 19 $(70 \%)$ and pyrexia in $17(63 \%)$. A factor predisposing to tuberculosis or a history of recent contact was found in $12(43 \%)$, and $21(75 \%)$ had positive cultures. Seventeen (61\%) had classical miliary shadowing while four $(14 \%)$ had cryptic miliary tuberculosis with no radiological evidence of tuberculosis. The remaining seven patients $(25 \%)$ had radiological changes consistent with pulmonary tuberculosis, but no miliary shadows. Of those who completed chemotherapy, only five $(42 \%)$ received 18 months' treatment. Nine patients $(32 \%)$ died from their miliary tuberculosis. Failure to consider the diagnosis, leading to a delay in starting chemotherapy, appeared to be a major problem.

Miliary or disseminated tuberculosis is the most severe form of tuberculosis, caused by haematogenous spread of tubercle bacilli. It used to be a disease of young children, occurring usually within a year of primary infection. ${ }^{\prime}$ It can also be a complication of any chronic tuberculous lesion in adults and with changes in the prevalence of tuberculosis the peak incidence of miliary spread was reported in the 1960 s to be in the eighth decade, the "cryptic" variety being as common as the "classical" presentation. ${ }^{2}$

We report our findings on miliary tuberculosis in South and West Wales from 1976 to 1978 as part of a retrospective survey of 1000 cases of tuberculosis in the area.

\section{Methods}

The patients with miliary tuberculosis were traced and documented as in our previous studies dealing with pulmonary and extrapulmonary tuberculosis. ${ }^{34}$ Additional information was extracted from the notes of patients with miliary tuberculosis including symptoms and signs at presentation, biochemical

Address for reprint requests: Dr RDH Monie, Southern General Hospital, Glasgow G51 4TF.

Accepted 7 January 1983 and haematological indices, the period of observation before the correct diagnosis was made, and abnormalities on the chest radiograph.

The cases were divided according to the radiographic appearances into (1) classical, if miliary shadows were present; (2) overt, if changes suggestive of active or previous tuberculosis (but not miliary shadows) were seen; and (3) cryptic, if there was no evidence on the radiograph to suggest tuberculosis.

\section{Results}

During the three years $28(2.8 \%)$ of the 1000 patients with tuberculosis reviewed had miliary spread. Seventeen $(61 \%)$ of these had miliary shadowing, seven $(25 \%)$ had other radiological changes consistent with active or previous pulmonary tuberculosis, and four (14\%) had no radiographic changes suggesting pulmonary tuberculosis.

The age distribution of the three groups is shown in table 1 . Only two of the 28 patients were less than 20 years of age while half were over 60 years. In the 11 patients with overt or cryptic disease only two were under the age of 60 years, while 12 of the 17 who had classical disease were under 60 . Sixteen patients $(57 \%)$ were male and six $(21 \%)$ were of non-European origin in a population where only about $2 \%$ are of non-European origin. ${ }^{5}$ 
Table 1 Classification of miliary tuberculosis according to age

\begin{tabular}{llll}
\hline Age $(y)$ & \multicolumn{3}{c}{ Type of miliary tuberculosis } \\
\cline { 2 - 4 } & Classical & Overt & Cryptic \\
\hline $0-9$ & 1 & & \\
$10-19$ & 1 & & \\
$20-29$ & 2 & 1 & \\
$30-39$ & 1 & & 1 \\
$40-49$ & 4 & 2 & 2 \\
$50-59$ & 3 & 3 & 1 \\
$60-69$ & 4 & 1 & \\
$70-79$ & 1 & $7(25 \%)$ & $4(14 \%)$ \\
80 and over & $17(61 \%)$ & 7 \\
Total &
\end{tabular}

\section{PREDISPOSING FACTORS}

Ten patients (36\%) had a factor predisposing to tuberculosis. Seven $(25 \%)$ were receiving corticosteroids or immunosuppressants and three were alcoholics. In addition, two children aged 9 and 14 years were diagnosed at routine screening after contact with cases of smear-positive pulmonary tuberculosis.

\section{CLINICAL FEATURES}

Presenting symptoms and signs were recorded in 27 patients. Nineteen $(70 \%)$ complained of anorexia and weight loss, $11(41 \%)$ had other constitutional symptoms, and on examination $17(63 \%)$ had a low-grade intermittent pyrexia. Nine patients had abdominal pain and a similar number complained of night sweats. Signs of disease of the central nervous system (altered level of consciousness, focal neurological deficit, choroidal tubercles, or meningism) were found in nine patients. Eight male patients had genitourinary tract symptoms, four having dysuria, frequency, or haematuria and four either epididymo-orchitis or a scrotal sinus. There was no difference in symptoms between any of the groups.

Two of the four patients with cryptic miliary tuberculosis presented with urinary symptoms and were found to have tubercle bacilli in urine and sputum. The other two patients died, one presenting with a pneumothorax and the other with meningitis.

\section{CULTURES}

Twenty-one patients $(75 \%)$ had positive cultures (table 2). Seventeen were diagnosed by repeated examination of sputum, gastric washings, urine, and cerebrospinal fluid, only four requiring more invasive procedures to make the diagnosis (pleural and bone marrow aspiration and lymph node and testicular biopsy). Eight patients (29\%) had positive cultures from two or more sites at the time of diagnosis.

In five of the remaining seven cases no specimens
Table 2 Sources of positive cultures from 28 patients with miliary tuberculosis

\begin{tabular}{llll}
\hline Culture & \multicolumn{3}{l}{ Type of miliary tuberculosis } \\
\cline { 2 - 4 } & Classical & Overt & Cryptic \\
\hline Sputum & 6 & 1 & \\
Gastric washings & 4 & 2 & 2 \\
$\begin{array}{l}\text { Urine } \\
\text { Cerebrospinal fluid }\end{array}$ & 1 & 1 & \\
$\begin{array}{l}\text { Pleural aspiration } \\
\text { Bone marrow }\end{array}$ & 1 & 1 & \\
Lymph node biopsy & 1 & & \\
Testicular biopsy & 1 & 1 & 1 \\
All cultures negative & 1 & 1 & 1 \\
Clinical diagnosis only & 1 & & 4 \\
Diagnosis at necropsy & 2 & 7 & \\
Total & 17 & & \\
\hline
\end{tabular}

were sent for culture-in two the diagnosis was based on clinical and radiological appearances and in three it was made only at necropsy. Only two patients, one with classical and one with cryptic miliary disease, had persistently negative cultures.

\section{HAEMATOLOGICAL INDICES}

Haematological indices were available for 23 patients. The haemoglobin concentration was normal (above $12 \mathrm{~g} / \mathrm{dl}$ ) in 11 patients while nine had a normochromic normocytic anaemia. Three patients (13\%) had macrocytosis-one was an alcoholic, another had acute lymphoblastic leukaemia, and the third was a patient taking the contraceptive pill who had a megaloblastic bone marrow. The white cell count was normal in 17 patients while four had a neutrophil leucocytosis (above $10 \times 10^{9} / \mathrm{l}$ ), and two were neutropenic, one with acute lymphoblastic leukaemia and the other with benign monoclonal gammopathy.

The erythrocyte sedimentation rate was variable, the fall in one hour being over $100 \mathrm{~mm}$ and under $20 \mathrm{~mm}$ in similar numbers of patients.

\section{BIOCHEMICAL INDICES}

Biochemical indices were available in 19 patients. The plasma urea concentration was raised in four patients $(21 \%)$ and the plasma sodium concentration was below $130 \mathrm{mmol}(\mathrm{mEq}) / 1$ in six $(31 \%)$, but no patient had a potassium concentration of less than $3.0 \mathrm{mmol}(\mathrm{mEq}) / 1$.

The serum albumin concentration was below 30 $\mathrm{g} / \mathrm{l}$ in eight patients $(42 \%)$ and six had a raised alanine aspartate transaminase concentration. Other liver function tests gave normal results.

\section{MANAGEMENT}

Four of the 28 patients complied badly with treatment, although three of them were thought to have taken drugs intermittently for at least nine months. 
Twelve patients completed treatment as instructed by the supervising physician, five $(42 \%)$ receiving it for 18 months and seven (58\%) for nine to 13 months. All patients had their treatment supervised by a chest physician. The remaining 12 patients died, nine from their tuberculosis.

\section{DEATHS}

Nine patients $(32 \%)$ died from their infection and had necropsies that confirmed the diagnosis.

Four $(21 \%)$ of the 17 patients with classical shadowing on their chest radiograph died. One, an alcoholic, was admitted in a moribund condition and died within 24 hours; in the second, consideration of an alternative diagnosis of left ventricular failure led to a delay of 19 days in making the correct diagnosis; and in the other two patients the diagnosis was made only at necropsy, the radiographic appearances during life being interpreted as signs of a connective tissue disorder and coalworkers' pneumoconiosis respectively.

Three of the seven patients $(43 \%)$ with overt radiological evidence of pulmonary tuberculosis (but not miliary shadowing) died. Two had pleural effusions but pleural fluid was never examined for tubercle bacilli. Treatment was started on the ninth and 49th days after admission but both patients died the following day. The third patient had bronchopneumonic shadowing with tubercle bacilli in the sputum but died 40 days after starting treatment from perforated tuberculous ulcers of the ileum.

Two of the four patients with cryptic miliary tuberculosis died. One presented with a pneumothorax and the diagnosis was made at necropsy; the other, who presented with meningitis, had biochemical changes in the cerebrospinal fluid suggestive of tuberculosis but died three days later, despite antituberculous treatment, from adrenal failure due to tuberculous lesions of the glands.

The length of observation before diagnosis was no greater in patients who died than in those who survived. Ten of the patients with classical miliary shadowing were observed for over seven days before starting treatment. In one patient with cryptic spread the period between onset of symptoms and diagnosis was two years.

RELAPSES

So far none of the 16 patients who survived have relapsed within the area surveyed.

\section{Discussion}

Miliary tuberculosis is no longer a common condition. Only $2.8 \%$ of 1000 patients with tuberculosis had evidence of disseminated spread, which for the population studied gives an incidence of 0.45 cases per 100000 population per year. The diagnosis of miliary tuberculosis was made in nine patients only at necropsy; in another 13 it was indicated by miliary shadowing on the chest radiograph and in the remaining six by simultaneous positive cultures from two sites.

Previous studies have emphasised the difficulties in diagnosing some cases of miliary tuberculosis and have referred to them as "concealed" or "cryptic." In our patients with miliary tuberculosis the most common symptoms and signs were weight loss and general malaise with a low-grade pyrexia. Respiratory symptoms were uncommon. Nine patients complained of abdominal pain, but in only three patients was hepatosplenomegaly recorded. Disease of the central nervous or genitourinary systems was seen in most patients. No differences in symptoms or signs were noted between any of the three groups of patients.

The age incidence of miliary tuberculosis has steadily risen: half the patients in this survey were over 60 years and most of these had overt or cryptic disease. It is thought that in the elderly reinfection can behave as primary infection because of an agerelated change in immunity. Elderly people, however, frequently have concomitant diseases which may predispose to activation of previously acquired infection.

Seven patients were receiving corticosteroids or immunosuppressants at the time of diagnosis. Millar and Horne noted suppression of symptoms when patients were on such drugs, leading to a delay in diagnosis of the tuberculosis. ${ }^{7}$ Three of these patients died from their tuberculosis and one from the underlying condition.

There were more deaths in our survey than were noted by Proudfoot $e t$ al, ${ }^{2}$ five of the nine patients who died having overt or cryptic disease. Three of the four patients with classical disease who died had had an alternative diagnosis based on the chest radiographic appearances and had received treatment for that disease. None of these patients had sputum examined for tubercle bacilli. Only two patients $(7 \%)$ had negative cultures for tubercle bacilli (table 2) - one had classical disease and negative cultures for sputum, gastric washings, laryngeal swabs, urine, and bone marrow and the other had cryptic disease. In contrast, a greater proportion of patients in Proudfoot's series had negative cultures, $25 \%$ requiring a therapeutic trial with specific antituberculous drugs to confirm the diagnosis. In five of our patients $(18 \%)$ no material was sent for tuberculous cultures. Failure to consider the diagnosis thus was a greater problem in 1976-8 than in 1954-67. 
Previous authors have pointed out that patients with miliary tuberculosis have abnormal haematological findings, especially those with cryptic disease, in which granulocytic disorders appear more common. ${ }^{8-10}$ Normochromic normocytic anaemia and neutrophilic leucocytosis, both very non-specific features of chronic disease, were seen but no cases of hypochromic anaemia were found. ${ }^{11}$ Two patients with macrocytosis and neutropenia had underlying primary haematological disorders and treatment for these conditions probably activated previously quiescent tuberculosis. The third patient with macrocytosis had a megaloblastic bone marrow thought to be due to an abnormality of folate metabolism caused by the contraceptive pill, ${ }^{12}$ although it might have been a result of her miliary tuberculosis. ${ }^{11}$ The wide variation of the erythrocyte sedimentation rate makes that test of little help in the diagnosis of miliary tuberculosis.

The bacillary population in miliary tuberculosis is high, but despite this and the absence of a controlled trial of short-course chemotherapy for miliary tuberculosis seven $(58 \%)$ of the 12 patients who completed their chemotherapy received it for 13 months or less. Pyrazinamide was given to only two patients, which is surprising as meningeal disease is common in miliary tuberculosis and this drug crosses the blood-brain barrier as effectively as does isoniazid. ${ }^{13}$

We would like to thank the many physicians and surgeons who allowed us access to the case notes of patients in their care; the medical records staff in the areas; the medical officers for environmental health, the Welsh Office, and Dr PA Jenkins of the Mycobacterium Reference Laboratory, Cardiff, for helping us to trace the cases of tuberculosis; and Mrs
KH Chaloner, Mrs P Davies, and Mrs CM Rowe for their secretarial help.

\section{References}

' Seal RME. The pathology of tuberculosis. Br J Hosp Med 1971;5:783-92.

${ }^{2}$ Proudfoot AT, Akhtar AJ, Douglas AC, Horne NW. Miliary tuberculosis in adults. Br Med J 1969;ii:2736.

${ }^{3}$ Monie RDH, Hunter AM, Rocchiccioli KMS, White JP, Campbell IA, Kilpatrick GS. A retrospective survey of the management of pulmonary tuberculosis in South and West Wales 1976-8. Br Med $J$ 1982;284:571-3.

${ }^{4}$ Monie RDH, Hunter AM, Rocchiccioli KMS, White JP, Campbell IA, Kilpatrick GS. A retrospective survey of the management of extrapulmonary tuberculosis (excluding miliary and meningeal) in South and West Wales 1976-8. Br Med J 1982;285:415-8.

${ }^{5}$ Office of Population Censuses and Surveys. Small area statistics (1971 census). London: HMSO, 1973-4.

${ }^{6}$ Medd WE, Hayhoe FGJ. Tuberculous miliary necrosis with pancytopenia. $Q J$ Med 1955;24:351-63.

${ }^{7}$ Millar JW, Horne NW. Tuberculosis in immunosuppressed patients. Lancet 1979;i:1176-8.

${ }^{8}$ Oswald NC. Acute tuberculosis and granulocytic disorders. Br Med J 1963;ii:1489-95.

${ }^{9}$ Rodin AE, Hnalko SI. Non-reactive tuberculosis. Can Med Ass J 1963;89:817-20.

${ }^{10}$ Morrow LB, Anderson RE. Acute tuberculosis in leukaemia. Arch Pathol 1965;79:484-93.

${ }^{11}$ Cameron SJ, Horne NW. The effect of tuberculosis and its treatment on erythropoiesis and folate activity. Tubercle 1971;52:37-48.

12 Stephens MEM, Craft I, Peters TJ, Hoffbrand AV. Oral contraceptives and folate metabolism. Clin Sci 1972;42:405-14.

${ }^{13}$ Barling RWA, Selkon JB. The penetration of antibiotics into cerebrospinal fluid and brain tissue. J Antimicrob Chemother 1978;4:203-27. 